\title{
Penile Implant
}

National Cancer Institute

\section{Source}

National Cancer Institute. Penile Implant. NCI Thesaurus. Code C93088.

An implanted device that enables the penis to become erect. 